\title{
Expected Performance of Vertex Reconstruction in the ATLAS Experiment at the LHC
}

\author{
Eva Bouhova-Thacker, Vadim Kostyukhin, Thomas Koffas, Wolfgang Liebig, \\ Maaike Limper, Giacinto Nicola Piacquadio, Kirill Prokofiev, Christian Weiser, and \\ Andreas Wildauer, on behalf of the ATLAS Collaboration
}

\begin{abstract}
In the harsh environment of the Large Hadron Collider at CERN (design luminosity of $10^{34} \mathrm{~cm}^{-2} \mathrm{~s}^{-1}$ ) efficient reconstruction of vertices is crucial for many physics analyses. Described in this paper is the expected performance of the vertex reconstruction used in the ATLAS experiment. The algorithms for the reconstruction of primary and secondary vertices as well as for finding photon conversions and vertex reconstruction in jets are described. The implementation of vertex algorithms which follows a very modular design based on object-oriented $\mathrm{C}++$ is presented. A user-friendly concept allows event reconstruction and physics analyses to compare and optimize their choice among different vertex reconstruction strategies. The performance of implemented algorithms has been studied on a variety of Monte Carlo samples and results are presented.
\end{abstract}

Index Terms-Data analysis, data reconstruction, high energy physics, pattern recognition, reconstruction algorithms, tracking, vertex detectors.

\section{INTRODUCTION}

$\mathbf{T}$ HIS paper describes vertex reconstruction in the ATLAS Experiment at the Large Hadron Collider (LHC) at the European Organization for Nuclear Research (CERN), Geneva, Switzerland. In anticipation of the first collisions at the LHC, all presented results are based on Monte Carlo data using the full simulation of the ATLAS detector. All results have been approved by the ATLAS Collaboration.

\section{THE ATLAS DETECTOR}

The ATLAS Detector [1] is a particle detector in operation at the LHC at CERN. It is a multi-purpose detector designed to cover a wide range of physics analyses. For vertex reconstruction, the Inner Detector (ID) is of most importance. The ATLAS ID consists of three subsystems which, from inside to outside, are:

Manuscript received June 05, 2009; revised November 05, 2009. Current version published April 14, 2010. W. Liebig was supported by the Netherlands Organization for Scientific Research (NWO); research grant VIDI 680.47.218.

E. Bouhova-Thacker is with Lancaster University, Lancaster LA1 4YB, U.K. (e-mail: bouhova@mail.cern.ch).

V. Kostyukhin is with Physikalisches Institut der Universität Bonn, 53115 Bonn, Germany (e-mail: Vadim.Kostioukhine@cern.ch).

T. Koffas and K. Prokofiev are with CERN, 1211 Genève 23, Switzerland (e-mail: Thomas.Koffas@cern.ch; kprok@mail.cern.ch).

W. Liebig and M. Limper are with Nikhef, 1098 XG Amsterdam, The Netherlands (e-mail: liebig@mail.cern.ch; Maaike.Limper@cern.ch).

G. N. Piacquadio and C. Weiser are with the Physikalisches Institut, AlbertLudwigs-Universität Freiburg, 79104 Freiburg, Germany (e-mail: giacinto.piacquadio@physik.uni-freiburg.de; Christian.Weiser@cern.ch).

A. Wildauer is with IFIC, 46071 Valencia, Spain (e-mail: andreas. wildauer@cern.ch).

Digital Object Identifier 10.1109/TNS.2010.2040752
- silicon pixel detector with three layers in the barrel and three layers in each end-cap; the resolution in the $R \phi$ direction of the module is $10 \mu \mathrm{m}$ and $115 \mu \mathrm{m}$ in the $z$ direction of the module;

- silicon microstrip detector (SCT) with four double layers in the barrel and nine double layers in each end-cap; the resolution in the $R \phi$ direction of the module is $17 \mu \mathrm{m}$ and $580 \mu \mathrm{m}$ in the $z$ direction of the module;

- transition radiation tracker with $\mathrm{e}^{ \pm}$identification and an $\mathrm{R} \phi$ resolution of approximately $130 \mu \mathrm{m}$.

The radial positions of the barrel detectors range from $50.5 \mathrm{~mm}$ to $122.5 \mathrm{~mm}$ for the pixel detector, from $299 \mathrm{~mm}$ to $514 \mathrm{~mm}$ for the silicon microstrip detector and from $563 \mathrm{~mm}$ to 1066 $\mathrm{mm}$ for the transition radiation tracker. The coverage in pseudorapidity $|\eta|$ is up to 2.5 for the silicon detectors and up to 2 for the transition radiation tracker. The inner detector provides at best $3+4+36$ measurements per charged particle trajectory, thus allowing for efficient reconstruction of tracks and vertices.

\section{LHC CONDITIONS AND VERTEX TOPOLOGIES}

The Large Hadron Collider is designed to reach a luminosity of $10^{34} \mathrm{~cm}^{-2} \mathrm{~s}^{-1}$ at a center-of-mass energy of $14 \mathrm{TeV}$. This is the so-called design (or high) luminosity regime. In the beginning, however, the LHC will be operated at lower luminosities and lower center-of-mass energies. One anticipated luminosity regime is $2 \cdot 10^{33} \mathrm{~cm}^{-2} \mathrm{~s}^{-1}$ which in LHC terms is referred to as low luminosity. The high and low luminosity regimes result in production of on average 4.6 to 24 proton-proton interactions per bunch crossing, respectively. Each signal event reconstructed in the ATLAS detector will thus be superimposed with several so-called minimum bias events. Compared to the signal events, the minimum bias collisions usually have lower track multiplicities and lower transverse momenta. For all the studies presented in this paper, a center-of-mass energy of $14 \mathrm{TeV}$ has been used. The luminosity regimes used (low, high, signal only) vary but will be clearly indicated for each result.

The collision of the two proton bunches in the LHC leads to several different vertex topologies as shown in Fig. 1. In a typical collision event, several primary vertices along the beam as well as decays of long-lived particles, photon conversions, vertices in jets and vertices from decay chains, are produced. The reconstruction of vertices from these processes, distinguished by their different topologies, forms a vital part of the data analysis.

The reconstruction of primary vertices is important for many physics studies, including searches for new particles, tagging 


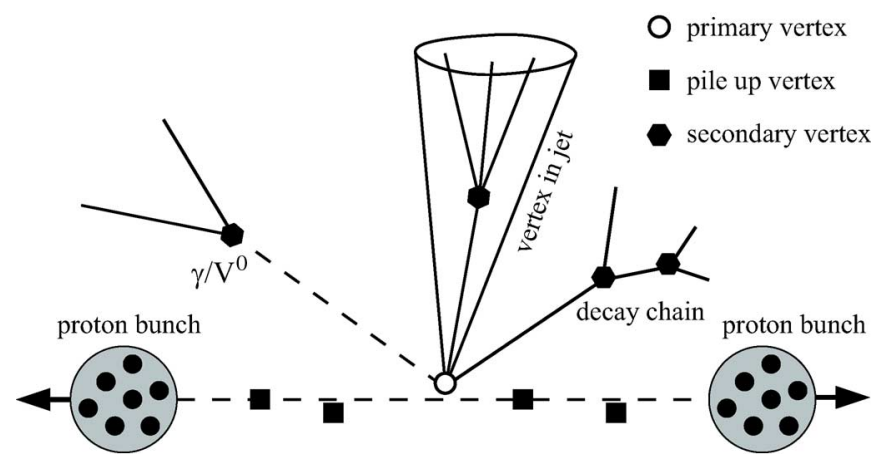

Fig. 1. Vertex topologies important for physics analyses in ATLAS: primary and pile up vertices, vertices from conversions and long-lived particles, vertices in jets and vertices from decay chains.

of $b$ - and $\tau$-jets, reconstruction of exclusive $B$-decays and similar applications. The correct identification of the primary vertex which stems from the hard inelastic collision is of particular importance in the LHC conditions. It is also important for determination of the LHC beam spot parameters.

The high accuracy of the silicon tracker will allow ATLAS to select jets from $b$-quarks by searching for tracks originating from a separate $b$-hadron decay vertex in the vicinity of the primary vertex. The efficient detection and reconstruction of such displaced vertices is essential to achieve a good $b$-tagging performance. However, the fragmentation of a $b$-quark results in a decay chain composed of a secondary vertex from the weakly decaying $b$-hadron and typically one or more tertiary vertices from $c$-hadron decays. The limited experimental resolution and high track density inside a jet therefore demand a dedicated vertex reconstruction, for which several approaches exist in ATLAS.

Particle decays in flight and even full decay chains are reconstructed using dedicated vertex finders. They exploit the assumed properties of the particle and the conservation laws governing its decay, and use them to apply additional kinematic constraints in the vertex fit.

About $40 \%$ of all photons produced in $p p$ collisions in ATLAS will convert in the material of the ID into $\mathrm{e}^{+} \mathrm{e}^{-}$pairs [1]. Hence, reconstruction of conversions is important for many physics analyses with photons in their final states. Reconstruction of conversions is also important to study the distribution of material in the detector (e.g. detector description in simulation, calibration of calorimeters).

The decay vertices of long-lived neutral particles $\left(\mathrm{V}^{0}\right.$ 's) are also reconstructed. This is useful for instance for $b$-tagging where tracks from $\mathrm{V}^{0}$ 's degrade the $b$-tagging performance due to their relatively large impact parameters. As in the case of secondary vertex reconstruction, these applications employ constrained vertex fitting.

\section{SOFTWARE FRAMEWORK}

The aim of the vertex reconstruction software in ATLAS is to provide a common and modular infrastructure to reconstruct the different vertex topologies discussed in the previous section. This is achieved by using a common event data model and common abstract interfaces based on object oriented $\mathrm{C}++$. The software for vertex reconstruction is fully integrated into the general ATLAS computing environment [2].

\section{A. Event Data Model}

The Event Data Model (EDM) defines the data classes in which information relevant for vertex reconstruction is stored. It is used to transfer information between algorithms and to store final results on disk. The EDM consists of classes representing reconstructed vertices and their relation to other objects used during a vertex reconstruction process (tracks, jets, etc.). Reconstruction of different vertex topologies requires different levels of detail concerning the data which needs to be stored in the various EDM classes. For instance, the reconstruction of primary vertices requires the storage of error matrices for each track. Reconstruction of the decay vertex of long-lived neutral particles however requires the correlations between tracks to be stored.

The flexibility of the level of details is achieved by using inheritance throughout the data model. This approach has two important advantages:

1) Quantities which are common to all vertex topologies are stored and retrieved the same way (common look and feel for users). This includes e.g. the vertex position and the vertex-track relations.

2) Only the required amount of detail for a given vertex topology is stored in the objects and hence on disk.

The EDM for vertex reconstruction also uses data classes of the general EDM for reconstruction of tracks whenever it has to deal with tracking quantities like track parameters or error matrices. End users and developers alike profit from this sharing of data classes.

\section{B. Interfaces}

Next to a common event data model, abstract interfaces are defined for all vertex reconstruction and related helper tasks. Algorithms for vertex reconstruction such as primary vertex finding or reconstruction of conversions make use of these interfaces to complete different steps of their reconstruction chain. Each interface can have several concrete implementations. The implementation used at each step is defined during run time by an external steering file.

An excerpt of various interfaces for vertex reconstruction is presented as follows:

- IVertexFitter: An interface for the implementation of vertex fitters. The fit method operates on a set of reconstructed tracks which it usually gets from a vertex finder. A starting point for the fit and additional vertex constraints (typically the beam spot) can also be used. The interface returns a single reconstructed vertex.

- IVertexFinder: An interface for the implementation of vertex finders. Concrete implementations analyze a track collection, pass a subset of tracks to a vertex fitter and return a set of reconstructed vertices (i.e. a vector of primary and pile up vertices).

- IVertexSeedFinder: An interface for the algorithms estimating the starting point of the vertex fit, given a set of tracks.

- IVertexLinearizedTrackFactory: An interface for storing the parameters of the linearized measurement equation, 


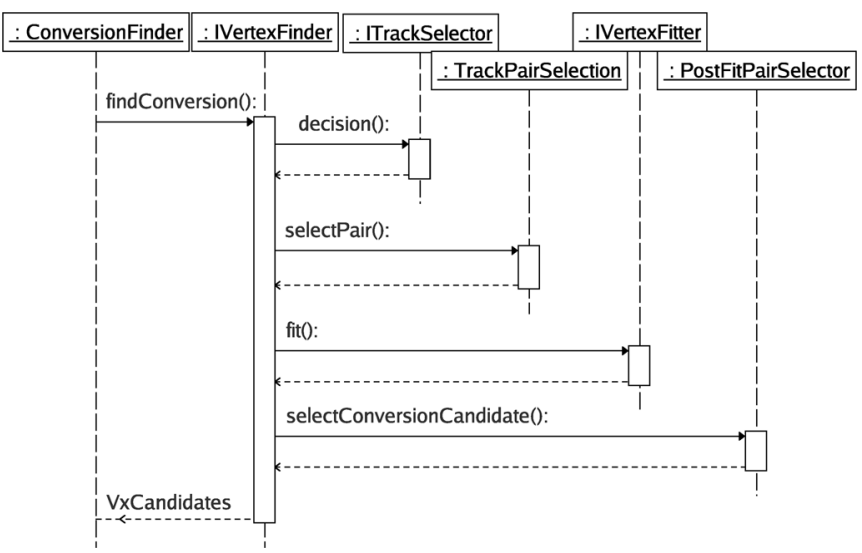

Fig. 2. Example sequence diagram showing the usage of abstract interfaces to define the reconstruction of conversions $\left(\gamma \rightarrow \mathrm{e}^{+} \mathrm{e}^{-}\right)$.

representing the dependence of the track parameters on the vertex position and on the track momentum at the vertex.

- IVertexUpdator: An interface for tools which are called iteratively to update the vertex estimate with one track at a time. Concrete implementations allow the addition or removal of a single track to or from a vertex candidate.

- IVertexSmoother: An interface for the implementation of algorithms which update the parameters of all tracks fitted to a vertex with the knowledge of the vertex position.

- ISecVertexInJetFinder: An interface for the reconstruction of secondary vertices in jets. As an input, the reconstructed primary vertex, the tracks associated to a jet and the jet direction as reconstructed in the calorimeter are provided, while the expected output is a set of one or more reconstructed displaced vertices.

- IVertexKinematicFitter: An interface for fitting a set of identified particles, i.e. tracks with associated particle masses, to a common vertex using a variable list of constraints.

- IKinematicConstraint: An interface for providing the kinematic vertex fitters with additional constraint equations. When invoked, each implementation supplies values and derivatives for a set of identified particles.

A simple use-case of these interfaces to define for example the reconstruction of conversions is given in Fig. 2. The so-called ConversionFinder algorithm uses (amongst others) concrete implementations of the IVertexFinder and IVertexFitter interfaces. The former object is responsible to find conversion candidates. It applies pre-selection cuts to a set of input tracks and retains pairs of oppositely charged tracks which are likely to originate from photon conversions. The latter object performs the actual vertex fit of conversion candidates and returns the fitted vertex. This procedure is often done in a loop where the actual fit gives feedback to the finder concerning the "quality" of the vertex fit. The finder then decides to go on or to accept the fitted vertex.

This structure of abstract interfaces, in conjunction with an event data model for vertex reconstruction, provides a common look and feel to the end-user who, regardless of the vertex topology, always works with the same EDM classes and interfaces. This design also allows for a high level of modularity and flexibility. An external python-based steering allows to exchange easily the concrete implementations of tools without updating the algorithms which use these implementations.

In addition, many different approaches can be applied and tested in parallel to achieve the best result for a certain vertex topology.

In the following sections, several vertex reconstruction chains which are based on these interfaces and event data model are presented. Their expected performance derived from Monte Carlo simulations are shown.

\section{RECONSTRUCTION OF PRIMARY VERTICES}

The reconstruction of primary vertices can generally be subdivided in two stages: primary vertex finding, dealing with the association of tracks to a particular vertex candidate, and vertex fitting, responsible for the reconstruction of the vertex position and corresponding covariance matrix. It is evident that since often these two processes are not easily distinguishable from each other, the "finding-through-fitting" and "fitting-after-finding" approaches are possible. The creation of separate base classes for algorithms responsible for vertex finding and fitting (Section IV-B) allow for modular implementation of both approaches in ATLAS.

One implementation in ATLAS, the so-called AdaptiveMultiVertexFinder (AMVF), is an example of the "finding-through-fitting" approach. The AMVF is based on an adaptive multi-vertex fitter [3]. The reconstruction starts with selection of tracks which are likely to originate from the interaction region. A single primary vertex candidate including all selected tracks is then formed and fitted. Tracks which are considered to be outliers during the first iteration of the fit are used to create a new vertex candidate. A simultaneous adaptive fit of two vertices is then performed. The number of vertex candidates grows in each iteration and these candidates compete with each other in order to gain more tracks. An annealing procedure prevents the finding procedure from falling into local minima.

An example of the "fitting-after-finding" approach in ATLAS is the so-called InDetPriVxFinder algorithm [4]. Here the reconstructed tracks compatible with the interaction region are pre-selected and the primary vertex candidates are formed by searching for clusters of tracks in the longitudinal projection. These clusters are then iteratively fitted with one available vertex fitter, rejecting outliers at every iteration. The maximal number of reconstructed vertices is thus fully determined at the seeding stage. Once a track is rejected from a vertex candidate, it is never used for any other cluster.

The variety of vertex fitters which can be used with InDetPriVxFinder includes the fast and full versions of the fitter proposed by Billoir [5]. They are denoted hereafter as Fast and Full fitters. A similar Billoir-based approach implemented in the stand-alone vertex fitter of the so-called VKalVrt package [6] can also be used.

Presented in Table I are the efficiencies to reconstruct and correctly identify the signal primary vertex from the process $W H, H\left(M_{H}=120 \mathrm{GeV}\right) \rightarrow b \bar{b}$ under low luminosity conditions (i.e. on average 4.6 minimum bias events superimposed). Efficiencies have been obtained by requiring that the true primary vertex is closer than $5 \mathrm{~mm}$ in the longitudinal $\mathrm{z}$ direction 
TABLE I

RECONSTRUCTION EFFECIENCIES AND RESOLUTIONS IN THE TRANSVERSE POSITIONS FOR SignAl PRIMARY VERTICES IN THE LOW LUMINOSITY REGIME OF THE LHC

\begin{tabular}{|c||c||c||c||c|}
\hline Efficiencies $(\%)$ & AMVF & VKalVrt & Fast fitter & Full fitter \\
\hline$W H, H(120) \rightarrow b \bar{b}$ & 95.35 & 96.25 & 89.38 & 89.39 \\
\hline Resolutions $(\mu \mathrm{m})$ & AMVF & VKalVrt & Fast fitter & Full fitter \\
\hline$W H, H(120) \rightarrow b \bar{b}$ & 11.21 & 11.34 & 12.69 & 12.69 \\
$W H, H(120) \rightarrow u \bar{u}$ & 10.02 & 10.03 & 10.68 & 10.68 \\
$H \rightarrow 4 l$ & 9.81 & 9.72 & 10.4 & 10.4 \\
$t \bar{t}$ & 10.33 & 10.33 & 12.55 & 12.55 \\
\hline
\end{tabular}

to the reconstructed primary vertex. The signal primary vertex in a bunch crossing is identified by selecting the primary vertex with highest weighted sum of the transverse momenta squared of used tracks. The statistical errors are below $1 \%$ in all the cases. It can be noted that the highest efficiencies are achieved by the AMVF and VKalVrt algorithms. Indeed, comparing to the other finders, these methods provide more robust approaches allowing the number of vertex candidates to be changed during the finding process and to deal efficiently with outlying tracks.

Also presented in Table I are the resolutions in the transverse coordinate of signal primary vertices reconstructed with the different approaches. Results are shown for the signal channels $W H, H(120) \rightarrow b \bar{b}, W H, H(120) \rightarrow u \bar{u}, H \rightarrow 4 l$ and $t \bar{t}$ with minimum bias events superimposed according to low luminosity conditions of the LHC. The resolutions are defined as standard deviations of Gaussians fitted to the corresponding distributions of residuals. It can be noted that all the resolutions of the transverse positions of reconstructed vertices are similar in all approaches: approximately $10 \mu \mathrm{m}$ to $12 \mu \mathrm{m}$. The calculated errors on the resolution values are typically smaller than $1 \mu \mathrm{m}$.

The resolutions on the longitudinal positions on primary vertices are typically of the order of $35 \mu \mathrm{m}$ to $55 \mu \mathrm{m}$, depending on the approach and the kinematics of the channel. The width on the pull distributions is around 1.08 for the transverse direction and around 1.19 on the longitudinal directions. The error on the width is less than 0.01 in all cases. Therefore, it can be noted that the residual distributions can reasonably well be approximated by a Gaussian and that the errors on the reconstructed vertex positions are correctly estimated for Monte Carlo.

The results of the AMVF and VKalVrt primary vertex algorithms are very comparable in the primary vertex selection efficiency and the resolutions in transverse and longitudinal directions. At the moment, the AMVF has been chosen as the default primary vertex finder to be used with low and high luminosity conditions and center-of-mass energies of $10 \mathrm{TeV}$ or higher. For the very early data with a center-of-mass energy of $900 \mathrm{GeV}$ and absence of pile up, the more robust Billoir fast or full fitters in conjunction with the InDetPriVxFinder can be used.

\section{VERTEX ReCONSTRUCTION With KinEMATIC CONSTRAINTS}

The reconstruction of vertices which stem from a heavy flavor decay, a converted photon or the decay of a long-lived hadron, usually involves the application of kinematic constraints in the vertex fit. Constrained vertex fitting is therefore used in several packages in the ATLAS software framework. Most commonly it

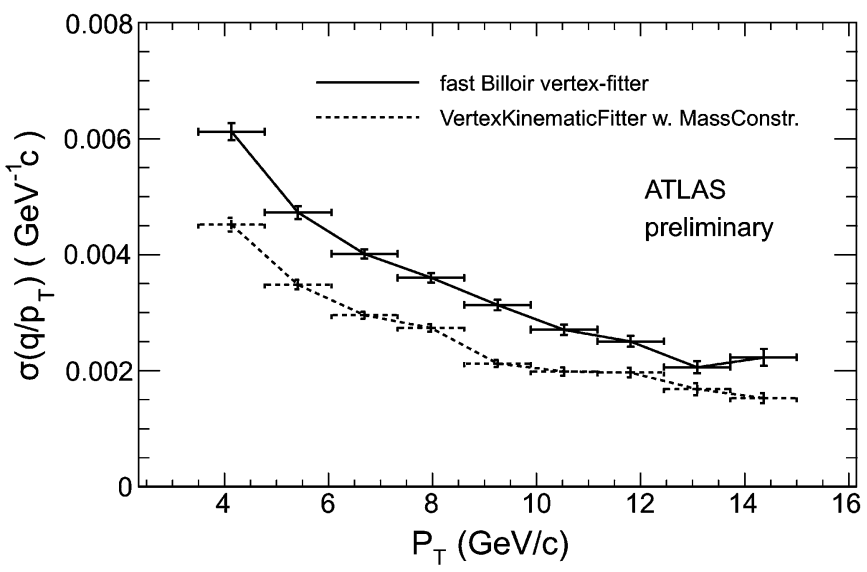

Fig. 3. Momentum resolution of the track parameters of muons from $J / \psi \rightarrow$ $\mu^{+} \mu^{-}$at the vertex vs. the transverse momentum of the $J / \psi$. The decay vertex was reconstructed with once with the fast Billoir vertex fitter and once with the VertexKinematicFitter including a mass constraint.

is implemented under an "all-in-one" fitting approach that carries specific applications of constraint vertex fitting out, such as the secondary vertex and decay chain fitting in the VKalVrt package [6]. The most useful type of constraint forces the mass of the decaying particle to the value corresponding to the particle hypothesis. In the case of a converted photon, the massless nature of the decaying particle allows the mass constraint to be applied directly as a collinearity constraint on the track parameters, leading to improved robustness of the fit convergence.

Recently, a new tool for constrained vertex fitting has been developed under the modular concept described in Section IV. This new tool, the so-called VertexKinematicFitter, is based on $\chi^{2}$ minimization with Lagrange multipliers, to simultaneously satisfy a vertex-constraint and any other kinematic constraint requested by the user. The Lagrange formalism allows each different constraint to be implemented as a separate class with a common abstract interface. An example of the use of the VertexKinematicFitter is given in Fig. 3, showing how the use of a mass-constraint in simulated $J / \psi \rightarrow \mu^{-} \mu^{+}$events improves the resolution of the momentum measurement (curvature) of the two emerging muon tracks. Since this is a very recent and new development it was decided to label the plot as "preliminary".

\section{ReCONSTRUCtion OF CONVERTED PHOtONS AND LONG-LIVED NEUTRAL PARTICLES}

The reconstruction of the decay of a long-lived particle or a converted photon vertex involves the application of a mass or angular constraint. The basic structure to reconstruct the vertex and apply a constraint is similar in both cases.

Fig. 2 illustrates the case of reconstructing the photon conversion. Here the basic components are: the track selection and subsequent track classification, the formation of pairs of tracks with opposite charge, the vertex fitting and reconstruction of photon conversion vertex candidates, and the final post-fit selection of the conversion candidates. Constrained vertex fitting can be very CPU-time consuming, therefore a careful pre-selection of track and pair candidates is necessary. In the case of converted photons, for instance, electron tracks are selected using the particle identification capabilities provided by the ATLAS 


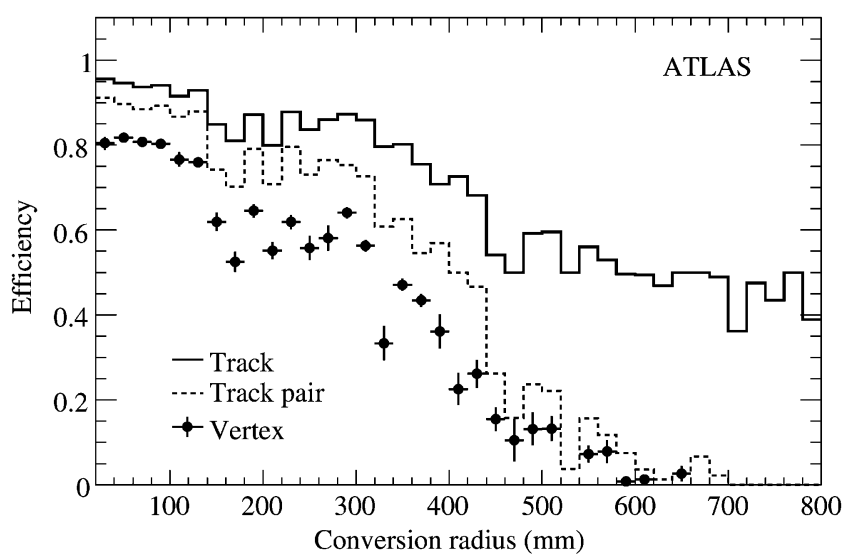

Fig. 4. Track, track-pair and vertex reconstruction efficiency for converted pho-

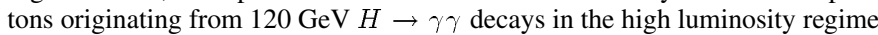
of the LHC. The efficiency is shown as a function of distance from the beam axis. Only tracks with $|\eta|$ smaller than 2 have been considered.

Transition Radiation Tracker (TRT). Track pairs are also pre-selected by requiring the two tracks of a converted photon to have small initial polar angle differences. In the case of both, converted photons and massive decaying particles, the distance of minimum approach between the two tracks in question has to be sufficiently small. In order to facilitate the convergence of the constrained fit, an initial estimate of the vertex position is also provided. This is done by computing geometrically the intersection point of two circles. Of the resulting two intersection points, the one with the closest approach along the beam-axis (z) is selected. The above pre-selection results in a significant reduction of the combinatorial background before the actual constrained vertex fit is performed. Additional reduction is achieved by exploiting the vertex fit results themselves. In the case of longlived decaying particles an additional step involving an un-constrained vertex fit is needed to reduce combinatorial background before the appropriate mass constraints are applied.

Only tracks originating from a radial distance from the beam axis of up to approximately $800 \mathrm{~mm}$ can be efficiently reconstructed by the tracking algorithm. However, the efficiency for reconstructing track pairs is significantly reduced at radial distances above $400 \mathrm{~mm}$. This is due to the missing measurements from the pixel detector and the reduced number of measurements in the silicon strip detector. The situation becomes even more pronounced in the case of high $\mathrm{p}_{\mathrm{T}}$ decaying particles. In addition, due to ATLAS tracker geometrical constraints, the track reconstruction efficiency is severely curtailed for pseudorapidity values of $|\eta|>2$, although tracks are still reconstructed up to $|\eta|=2.5$. In Fig. 4 the reconstruction efficiencies for converted photons originating from a $120 \mathrm{GeV} H \rightarrow \gamma \gamma$ decay in the high luminosity regime of the LHC are shown. A geometrical acceptance cut of $|\eta|<2$ has been applied on all tracks.

The resolution of the reconstructed radial position of the vertex for the case of converted photons originating from 120 $\mathrm{GeV}$ Higgs boson decays in the high luminosity regime of the LHC is shown in Fig. 5. The constrained vertex fit, requiring tracks to have the same direction at the vertex, has been used. The long tail to the right is due to bremsstrahlung losses of the two produced electron tracks, which reduce the individual track

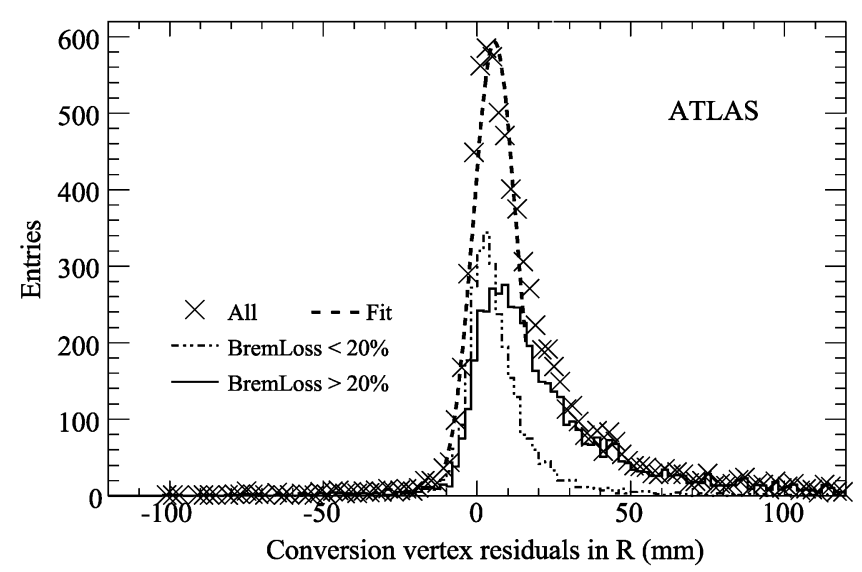

Fig. 5. Reconstructed vertex radial position resolution (in $\mathrm{mm}$ ) for converted photons from a $120 \mathrm{GeV} H \rightarrow \gamma \gamma$ decay. For comparison the two cases where the participating tracks have lost $>20 \%(<20 \%)$ of their energy due to bremsstrahlung are also shown separately. Only the Gaussian-like core of the distribution is fitted.

parameter reconstruction quality, hence severely affecting also the vertex fit results. To illustrate this particular point, the radial position resolution, with/without significant (greater than 20\%) losses due to bremsstrahlung, is also plotted separately. Clearly the tail is reduced when both contributing electrons have lost less than $20 \%$ of their energy due to bremsstrahlung effects. For the moment no bremsstrahlung energy loss corrections are applied, although work is progressing well towards achieving that.

An overall radial position resolution of approximately $7 \mathrm{~mm}$ has been achieved. There is still a bias of approximately $6 \mathrm{~mm}$, primarily due to the existence of the long bremsstrahlung induced tail. No effort to correct for energy losses of this type has been applied at present. The fact that the photon is a massless particle, resulting in an extremely small angular opening of the emitted tracks, makes it more difficult to reconstruct accurately the position of the conversion vertex. Position resolution is of the highest importance, since the reconstructed converted photons can offer the means to accurately map the ATLAS tracker material using minimum bias data. For more details on the conversion reconstruction and their applications see [7].

Bremsstrahlung losses on the other hand are not present in the case of the $K_{S} \rightarrow \pi^{+} \pi^{-}$decay. This, as well as the non-zero opening angle, provide a better test scenario for constrained vertex fitting. As mentioned above, instead of the angular constraint used in the case of the photon conversions, a direct mass constraint is implemented. In Fig. 6 the resolution of the reconstructed radial position for $K_{S}$ decays in $B_{d} \rightarrow J / \psi K_{S}$ events (without any minimum bias events superimposed) is shown. The absence of a bremsstrahlung related tail compared to that in Fig. 5 is evident. The overall radial position resolution, obtained from a Gaussian fit to the core of the distribution, is approximately $0.3 \mathrm{~mm}$. The variation of the radial position resolution with the distance from the beam axis is shown in Fig. 7 for the case of $K_{S}$ decays. The overall resolution degrades at higher radial distances where measurements of the high precision pixel and silicon-strip tracker components are fewer or missing. The resolution is better the closer the vertex position is to a given 


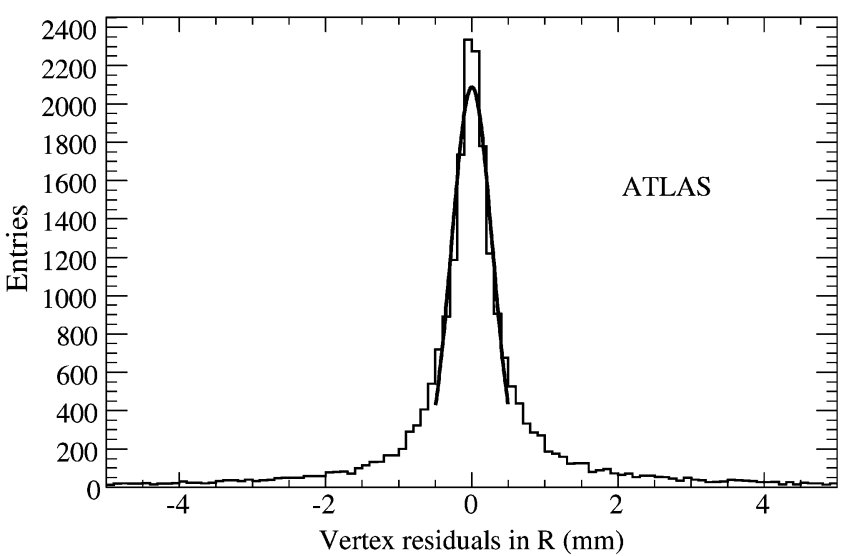

Fig. 6. Reconstructed vertex radial position resolution (in $\mathrm{mm}$ ) of $K_{S} \rightarrow$ $\pi^{+} \pi^{-}$decays.

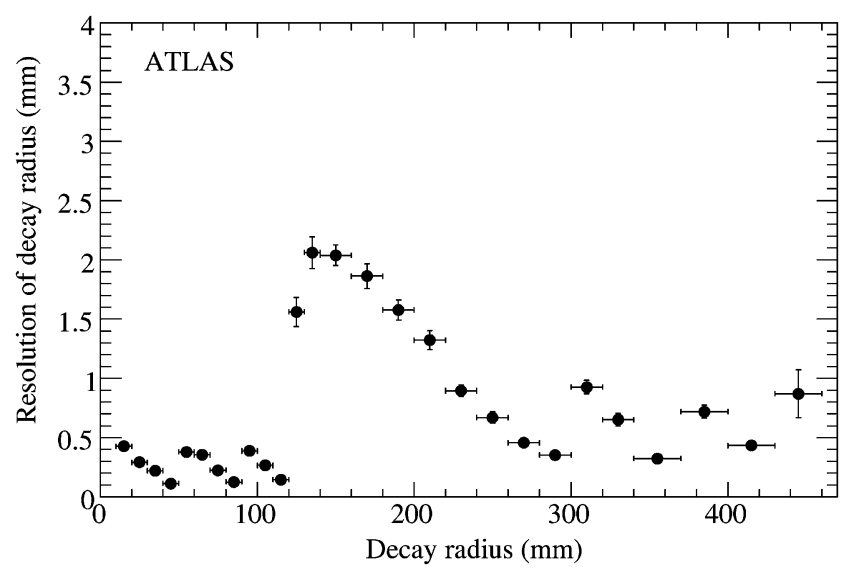

Fig. 7. Reconstructed vertex radial position resolution (in $\mathrm{mm}$ ) of $K_{S} \rightarrow$ $\pi^{+} \pi^{-}$decays as a function of the radial distance from the beam axis.

layer and is worst immediately after that. The degradation is proportional to the distance between layers. The minima correspond to the radial positions of the barrel layers. The largest increase at approximately $115 \mathrm{~mm}$ is due to the $200 \mathrm{~mm}$ gap between the Pixel and the Silicon Strip Trackers. Similar behavior is exhibited by the conversion radial position resolution, albeit with a certain amount of deterioration due to the bremsstrahlung losses, as discussed above.

\section{RECONSTRUCTION OF VERTICES IN JETS}

The most basic $b$-tagging algorithms in ATLAS rely directly on the impact parameter significance of the charged particles associated to the jet. These impact parameters are computed with respect to the reconstructed primary vertex (PV). The detection and reconstruction of one or more secondary vertices in a $b$-jet can considerably improve the $b$-tagging performance in two main aspects:

- Tracks originating from the decay of long-lived particles ( $K_{s}, \Lambda$, photon conversions) can be efficiently rejected.

- The kinematic properties of the $b$ decay and $b \rightarrow c$-decay vertices can be used as an additional handle against badly reconstructed tracks in light-quark jets.

Two main vertex finding algorithms for $b$-tagging are implemented in ATLAS. The first one relies on the assignment of all displaced tracks to a common geometrical vertex (inclusive vertex finder). The second one relies on the identification of the $P V \rightarrow b \rightarrow c$ decay chain topology (topological vertex finder). The inclusive vertex finder is based on a vertex fitting method proposed by Billoir [5] and is implemented in the VKalVrt package [6]. The topological vertex finder uses an extension of the Kalman Filter formalism for vertex reconstruction developed in ATLAS and is implemented in the JetFitter package [8].

The initial finding strategy is common for both finders:

- Selection of displaced tracks;

- Reconstruction of all 2-track vertices;

- Removal of vertices which are compatible with $\gamma, K_{S}$ and $\Lambda$ decay hypotheses.

The inclusive vertex finder then tries to reconstruct a common geometrical vertex out of the surviving tracks. Tracks with a bad $\chi^{2}$ contribution are removed iteratively from the fit, until the overall $\chi^{2}$ is below a predefined threshold. The vertex $\chi^{2}$ probability is not expected to follow a flat distribution, because of the assumption of a single geometrical vertex. A tuning of this threshold to achieve the best efficiency to purity ratio is thus required.

The topological vertex finder solves the pattern recognition problem by relying on the assumption that all tracks intersect a common PV $\rightarrow b$ - hadron $\rightarrow c$ - hadron flight axis, thus reducing a three-dimensional clustering problem to a onedimensional one:

1) A first fit is performed, initializing the $b$-flight axis with the calorimetric jet direction and assuming that all tracks form single vertices. This determines the $b$-flight axis direction and its intersections with the single tracks.

2) The compatibility between all pairs of vertices is evaluated by computing for each pair the overall $\operatorname{Prob}\left(\chi^{2}\right)$ of the decay chain fit after the two vertices have been merged.

3) The pair of vertices with the highest compatibility is clustered together into a single vertex and the decay chain fit is repeated.

4) The merging procedure is repeated iteratively starting again from (2) until no pair of vertices is compatible by more than $\operatorname{Prob}\left(\chi^{2}\right)=0.1 \%$ anymore.

This procedure results in a well defined vertex topology, with the advantage that also single-prong decays (i.e. vertices with a single reconstructed track) can be accessed.

In Tables II and III the performance of the two different strategies on jets selected from a sample of $W H, H(120) \rightarrow b \bar{b}, c \bar{c}$ or $l \bar{l}$ Monte Carlo events (with no minimum bias events superimposed) are presented. In the first table the vertex reconstruction efficiency for jets of transverse momenta between 50 and $80 \mathrm{GeV}$ is shown. In $b$-jets both algorithms reconstruct one or more vertices in 70-75\% cases: while the inclusive vertex finder can only assess the presence of a single inclusive vertex, the topological vertex finder can reconstruct a variety of different decay chain topologies. In particular, when the experimental resolution is sufficient with respect to the distance between the weak $b$ - and $c$-hadron decay positions, the topologies with two single-prong vertices (indicated for brevity in the table as single tracks), with a vertex (with two or more associated tracks) plus a single prong-vertex and with two vertices (with two or more 
TABLE II

VERTEX RECONSTRUCTION EFFICIENCY IN $b$-, $c$ - AND Light-JETS

\begin{tabular}{|c||c||c||c||c|}
\hline 1 Algorithm & Topology & $b$ & $c$ & 1 \\
\hline Inclusive finder & Nothing & 13.5 & 68.0 & 93.3 \\
& 1 Inclusive Vertex & 76.5 & 32.0 & 6.7 \\
\hline Topological finder & Nothing & 13.7 & 51.3 & 79.7 \\
& 1 Single Track & 9.9 & 17.4 & 13.6 \\
& 2 Single Tracks & 4.5 & 2.6 & 1.0 \\
& 1 Single Vertex & 49.6 & 25.1 & 5.2 \\
& 1 Vertex + 1 Track & 15.9 & 3.1 & 0.4 \\
& 2 Vertices & 6.3 & 0.5 & 0.04 \\
\hline
\end{tabular}

TABLE III

TRACK-TO-VERTEX ASSOCIATION EFFICIENCY AND PURITY

\begin{tabular}{|c||c||c||c|}
\hline Finding algo. & Topology & Efficiency & Purity \\
\hline Inclusive & 1 inclusive B/D vertex & $69 \%$ & $92 \%$ \\
\hline Topological & 1 vertex & $74 \%$ & $91 \%$ \\
& 1 vertex +1 track & $80 \%$ & $85 \%$ \\
& 2 vertices & $85 \%$ & $89 \%$ \\
\hline
\end{tabular}

associated tracks) get accessible. In case that a vertex is found, the inclusive vertex finder correctly associates to it $69 \%$ of all well-reconstructed tracks from a heavy flavour decay, keeping at the same time a very high purity: $92 \%$ of the tracks at vertex come from $b$ - or $b \rightarrow c$ decays. The topological vertex finder correctly associates to the fitted decay chain a larger fraction of the reconstructed tracks produced in $b$ - or $c$-hadron decay. This is particularly true when a complex topology beyond the single vertex has been detected, however at the cost of slightly reduced purity. The vertices reconstructed in light-jets can be explained by a combination of badly measured tracks and real displaced tracks originating from conversions, hadronic interactions, $K_{S}$ or $\Lambda$ decays.

Shown in Fig. 8 is the resolution achieved on the inclusive $b$-hadron decay vertex with respect to the true $b$-hadron position in the transverse plane (in the direction of flight of the $b$-hadron). The core resolution is about $400 \mu \mathrm{m}$, with a big tail due to the contribution of tracks from $c$-hadron decays.

The vertices reconstructed by the vertex finder are then used to compute the variables important to the secondary vertexbased $b$-tagging algorithm:

- fraction of energy from charged particles at the decay vertex (vertices);

- invariant mass;

- number of tracks fitted to a vertex (vertices);

- presence of a reconstructed secondary vertex topology.

The information above is combined into a likelihood function. The correlation terms are normally not taken into account. In the case of the inclusive finder however, the correlations between the invariant mass and the fraction of energy from charged particles at the decay vertex are used via a two-dimensional probability density function (PDF). The templates for the PDFs in the likelihood function are obtained from a very large number of Monte Carlo events, including $t \bar{t}, t \bar{t} j j$ and $W H$ events. Both secondary vertex-based algorithms are then combined with the

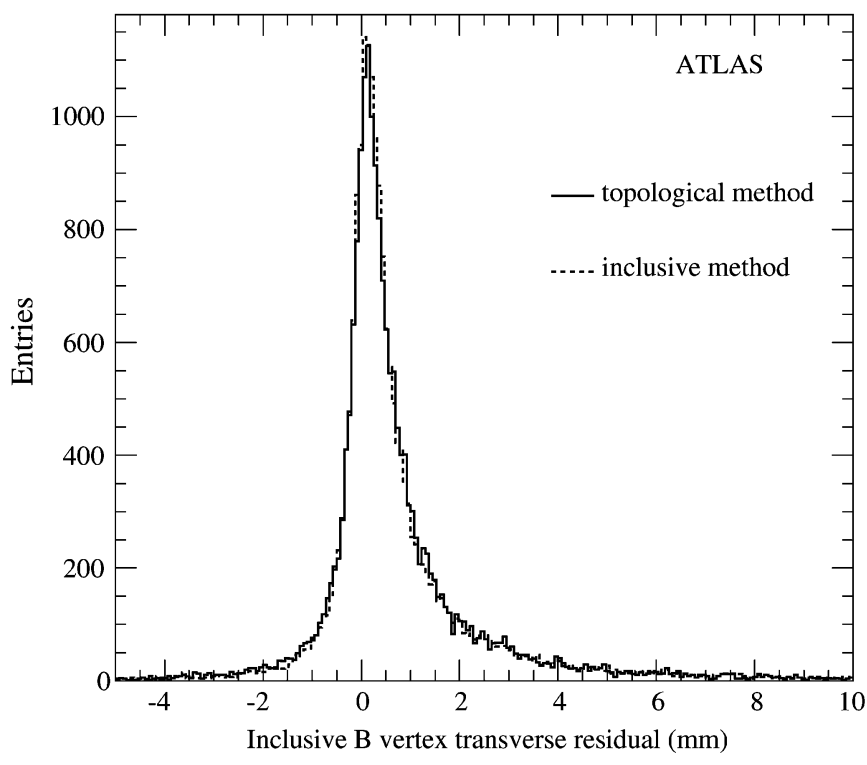

Fig. 8. Radial position resolution (in $\mathrm{mm}$ ) of the secondary vertex, for the inclusive vertex finder (BTagVrtSec in the figure) and for the topological vertex finder (JetFitter in the figure). In the latter case, the weighted average of all vertex positions in the decay chain is considered if more than one displaced vertex is found.

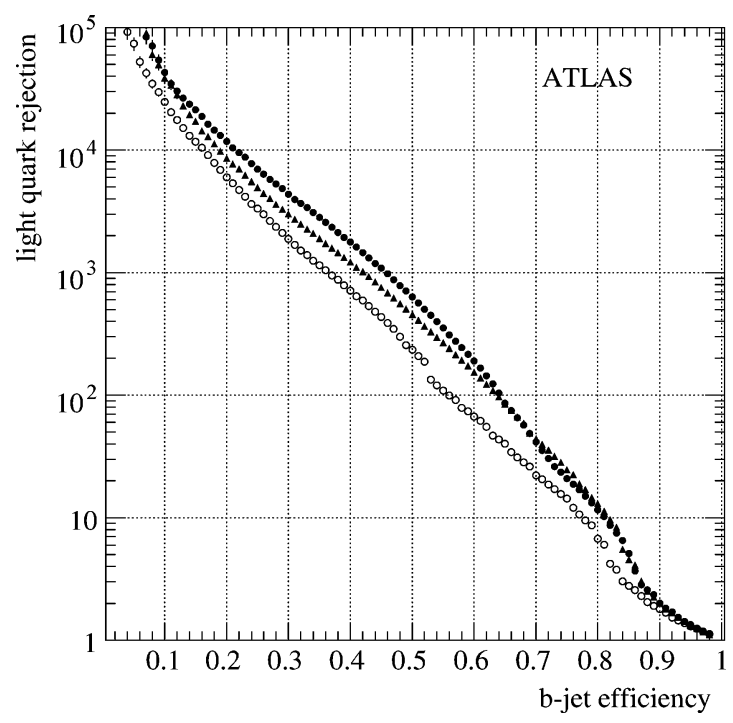

Fig. 9. Light quark rejection as a function of $b$-tagging efficiency for various algorithms: the inclusive vertex finder (triangles), the topological vertex finder (full circles) and, for comparison, the impact parameter only based algorithm (empty circles).

impact parameter-based tagging method, under the assumption that the two likelihood functions are uncorrelated.

The $b$-tagging performance achieved on $t \bar{t}$ and $t \bar{t} j j$ Monte Carlo events (with no minimum bias events superimposed) by the two secondary vertex-based algorithms is illustrated in Fig. 9. A comparison with the impact parameter-based algorithm is also presented. The rejection of light-quark jets is defined as the inverse of the probability to (mis)identify a light-jet as a $b$-jet. It can be noted that the use of the secondary vertex-based algorithms results in a significant improvement of the light-quark rejection. 


\section{SUMMARY}

Presented in this paper is the vertex reconstruction in the ATLAS Experiment at the Large Hadron Collider at CERN. An overview of the expected performance of primary vertex reconstruction, reconstruction of photon conversions and long-lived particle decays, constrained vertex fitting and vertex finding in jets for $b$-tagging is given. The algorithms and the underlying modular software environment are presented.

The vertex reconstruction has been tested extensively under as realistic conditions as possible using Monte Carlo datasets in anticipation of first collisions at the LHC. The simulation of these data sets includes the low and high luminosity regimes of the LHC and a displaced proton-proton collision point. It has been demonstrated that the vertex algorithms perform well under these conditions and that the vertex reconstruction software in ATLAS is well prepared for first data.

\section{ACKNOWLEDGMENT}

The authors would like to thank the ATLAS Inner Detector community who helped developing and testing the vertex reconstruction software. In particular we would like to thank
K. Grybel (Universitaet Siegen, Germany) for his help in producing some of the simulation data sets and H. Zhu (University of Sheffield, UK) for his contribution to Fig. 5.

\section{REFERENCES}

[1] G. Aad et al., The ATLAS Collaboration, "The ATLAS experiment at the CERN large hadron collider," J. Instrum., vol. 3, p. S08003, 2008.

[2] The ATLAS Collaboration, ATLAS Computing Tech. Design Rep. ATLAS TDR-017, CERN-LHCC-2005-022.

[3] W. Waltenberger and R. Fruhwirth, "Adaptive multi-vertex fitting," in Proc. Int. Conf. Computing in High Energy Physics, Interlaken, Switzerland, Sep. 27-Oct. 12004.

[4] N. G. Piacquadio, K. Prokofiev, and A. Wildauer, "Primary vertex reconstruction in the ATLAS experiment at LHC," in Proc. Int. Conf. Computing in High Energy Physics, Victoria, BC, Canada, Sep. 2-7, 2007.

[5] P. Billoir and S. Qian, Nucl. Instrum. Methods Phys. Res. A, vol. A311, pp. 139-150, 1992.

[6] V. Kostyukhin, VKalVrt-A Package for Vertex Reconstruction in ATLAS, ATL-PHYS-2003-031, 2003.

[7] The ATLAS Collaboration, Expected Performance of the ATLAS Experiment, Detector, Trigger and Physics, Geneva, Switzerland, 2008, CERN-OPEN-2008-020.

[8] G. Piacquadio and C. Weiser, "A new inclusive secondary vertex algorithm for $b$-jet tagging in ATLAS," J. Phys., Conf. Ser., vol. 119, p. 032032, 2008. 